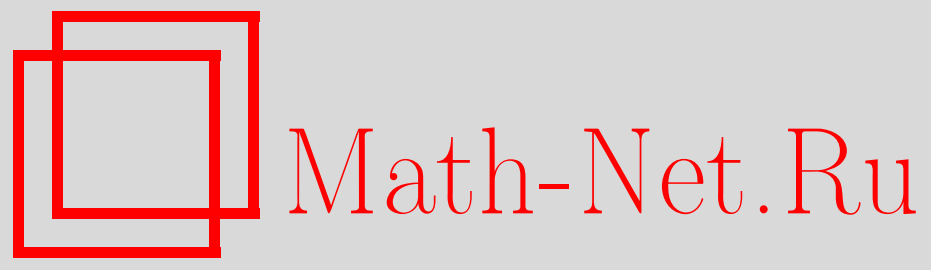

J. Kallsen, A. N. Shiryaev, Time Change Representation of Stochastic Integrals, Теория вероятн. и ее примен., 2001, том 46, выпуск 3, 579-585

DOI: https://doi.org/10.4213/tvp3906

Использование Общероссийского математического портала Math-Net.Ru подразумевает, что вы прочитали и согласны с пользовательским соглашением

http://www . mathnet.ru/rus/agreement

Параметры загрузки:

IP : 52.6 .47 .48

26 апреля 2023 г., $17: 37: 10$

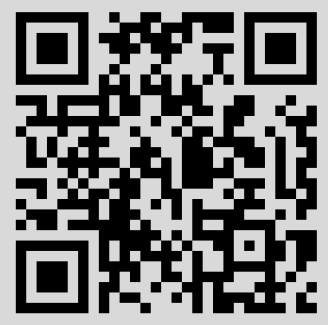


16. Золотарев B. M. Современная теория суммирования независимых случайных величин. М.: Наука, 1986.

Поступила в редакцию 30.VI.1998

(C) $2001 \Gamma$.

KALLSEN J. ${ }^{*}$, SHIRYAEV A. N.**

\section{TIME CHANGE REPRESENTATION OF STOCHASTIC INTEGRALS ${ }^{1)}$}

По теореме Дамбиса и Дубинса-Шварца любой стохастический интеграл $M=\left(\int_{0}^{t} H_{s} d W_{s}\right)_{t \in \mathbf{R}_{+}}$по броуновскому движению может быть записан как броуновское движение со случайной заменой времени, т.е. $M=\left(\widehat{W}_{\widehat{T}_{t}}\right)_{t \in \mathbf{R}_{+}}$для некоторого броуновского движения $\left(\widehat{W}_{\theta}\right)_{\theta \in \mathbf{R}_{+}}$и некоторой замены времени $\left(\widehat{T}_{t}\right)_{t \in \mathbf{R}_{+}}$. В [7] и [5] показано, что в этом утверждении броуновское движение можно заменить на (симметричное) $\alpha$-устойчивое дөижение Леви. Используя процесс кумулянт семимартингала, мы даем короткие новые доказательства. Кроме того, мы показываем, что это утверждение не может быть распространено на другие процессы Леви.

Ключевые слова и фразы: устойчивые движения Леви, кумулянтный процесс, стохастический интеграл, замена времени.

1. Time change representations. We generally use the notation of [2]-[4]. The transposed of a vector or matrix $x$ is denoted as $x^{\top}$ and its components are denoted by superscripts. Stochastic and Stieltjes integrals are written as $\left(H \cdot X_{t}\right)=\int_{0}^{t} H_{s} d X_{s}$. Increasing processes are identified with their corresponding Lebesgue-Stieltjes measure.

Let $\left(\Omega, \mathscr{F},\left(\mathscr{F}_{t}\right)_{t \in \mathbf{R}_{+}}, \mathbf{P}\right)$ be a filtered probability space as in [4, Definition I.1.2]. By Lévy process we refer to a process with stationary independent increments in the sense of $[4$, II.4.1]. Similar to [8, Definition 7.5.1], we make the following

$\mathrm{D}$ e f i $\mathrm{n}$ i t i o $\mathrm{n} 1$. Let $\alpha \in(0,2]$. An $\alpha$-stable Lévy motion is a Lévy process $X$ such that $X_{1}$ (or equivalently any $X_{t}$ ) has a strictly $\alpha$-stable distribution (i.e., $X_{1} \sim$ $S_{\alpha}(\sigma, \beta, \mu)$ for some $\alpha \in(0,2] \backslash\{1\}, \sigma \in \mathbf{R}_{+}, \beta \in[-1,1], \mu=0$ or $\alpha=1, \sigma \in \mathbf{R}_{+}$, $\beta=0, \mu \in \mathbf{R}$ ). We call $X$ a symmetric $\alpha$-stable Lévy motion if the distribution of $X_{1}$ (or equivalently any $\left.X_{t}\right)$ is even symmetric $\alpha$-stable (i.e., $X_{1} \sim S_{\alpha}(\sigma, 0,0)$ for some $\alpha \in(0,2]$, $\sigma \in \mathbf{R}_{+}$).

$\mathrm{D}$ e f $\mathrm{i} \mathrm{n}$ i $\mathrm{t}$ i o $\mathrm{n}$ 2. 1. A time change is a right-continuous increasing $[0, \infty]$-valued process $\left(T_{\theta}\right)_{\theta \in \mathbf{R}_{+}}$such that $T_{\theta}$ is a stopping time for any $\theta \in \mathbf{R}_{+}$. It is called finite if $T_{\theta}<\infty \mathbf{P}$-almost surely for any $\theta \in \mathbf{R}_{+}$.

2. By $\widehat{\mathscr{F}}_{\theta}:=\mathscr{F}_{T_{\theta}}$ we define the time-changed filtration $\left(\widehat{\mathscr{F}}_{\theta}\right)_{\theta \in \mathbf{R}_{+}}$.

3. The inverse time change $\left(\widehat{T}_{t}\right)_{t \in \mathbf{R}_{+}}$is defined as $\widehat{T}_{t}:=\inf \left\{\theta \in \mathbf{R}_{+}: T_{\theta}>t\right\}$.

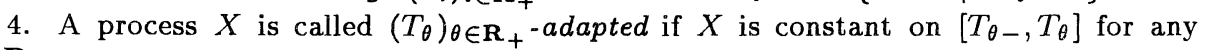
$\theta \in \mathbf{R}_{+}$.

R. e m a r k. If $X$ is a semimartingale on $\left(\Omega, \mathscr{F},\left(\mathscr{F}_{t}\right)_{t \in \mathbf{R}_{+}}, \mathbf{P}\right)$ and $\left(T_{\theta}\right)_{\theta \in \mathbf{R}_{+}}$is a finite time change, then the process $\left(\widehat{X}_{\theta}\right)_{\theta \in \mathbf{R}_{+}}$defined by $\widehat{X}_{\theta}:=X_{T_{\theta}}$ is a semimartingale

* Universität Freiburg, Institut für Mathematische Stochastik, Eckerstr., 1, D79104 Freiburg, Germany; e-mail: kallsen@neyman.mathematik.uni-freiburg.de

** Математический институт им. В. А. Стеклова РАН, ул. Губкина, 8, 117966 Москва, ГСП-1, Россия; e-mail: shiryaev@mi.ras.ru

1) Работа второго автора поддержана Alexander von Humboldt Foundation и Российским фондом фундаментальных исследований (грант № 99-01-00152). 
on $\left(\Omega, \mathscr{F},\left(\widehat{\mathscr{F}}_{\theta}\right)_{\theta \in \mathbf{R}_{+}}, \mathbf{P}\right)$ (cf., e.g., [2, (10.12)]). Many other properties are generally only

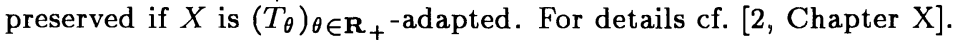

We are concerned with the following representation property which follows from the Dambis and Dubins-Schwarz theorem (cf. [6, Theorem V.1.6]).

Theorem 1. Let $X$ be 'a standard Brownian motion. Moreover, let $M:=H \cdot X$ for some $H \in L(X)$ ([2], [3]) such that $\int_{0}^{t} H_{s}^{2} d s \rightarrow \infty$ for $t \rightarrow \infty$. Then there exist a filtration $\left(\widehat{\mathscr{F}}_{\theta}\right)_{\theta \in \mathbf{R}_{+}}$on $(\Omega, \mathscr{F})$, a process $\left(\widehat{W}_{\theta}\right)_{\theta \in \mathbf{R}_{+}}$, and a finite time change $\left(\widehat{T}_{t}\right)_{t \in \mathbf{R}_{+}}$ such that

1) $\widehat{W}$ is a $\left(\widehat{T}_{t}\right)_{t \in \mathbf{R}_{+}}$-adapted Brownian motion on $\left(\Omega, \mathscr{F},\left(\widehat{\mathscr{F}}_{\theta}\right)_{\theta \in \mathbf{R}_{+}}, \mathbf{P}\right)$,

2) $M=\left(\widehat{W}_{\widehat{T}_{t}}\right)_{t \in \mathbf{R}_{+}}$.

If we define the $\left(\Omega, \mathscr{F},\left(\mathscr{F}_{t}\right)_{t \in \mathbf{R}_{+}}, \mathbf{P}\right)$-time change $\left(T_{\theta}\right)_{\theta \in \mathbf{R}_{+}}$by $T_{\theta}:=\inf \left\{t \in \mathbf{R}_{+}\right.$: $\left.\int_{0}^{t} H_{s}^{2} d s>\theta\right\}$, then we may choose $\widehat{\mathscr{F}}_{\theta}=\mathscr{F}_{T_{\theta}}, \widehat{W}_{\theta}=M_{T_{\theta}}$ and $\widehat{T}$ as the inverse time change of $T$. In particular, $\widehat{T}_{t}=\int_{0}^{t} H_{s}^{2} d s$ for $t \in \mathbf{R}_{+}$.

The previous theorem can be generalized to symmetric $\alpha$-stable Lévy motions (cf. [7] and [5]).

Theorem 2. Let $X$ be a symmetric $\alpha$-stable Lévy motion. Moreover, let $M:=H \cdot X$ for some $H \in L(X)$ such that $\int_{0}^{t}\left|H_{s}\right|^{\alpha} d s \rightarrow \infty$ for $t \rightarrow \infty$. Then there exist a filtration $\left(\widehat{\mathscr{F}}_{\theta}\right)_{\theta \in \mathbf{R}_{+}}$on $(\Omega, \mathscr{F})$, a process $\left(\widehat{L}_{\theta}\right)_{\theta \in \mathbf{R}_{+}}$and a finite time change $\left(\widehat{T}_{t}\right)_{t \in \mathbf{R}_{+}}$such that

1) $\widehat{L}$ is a $\left(\widehat{T}_{t}\right)_{t \in \mathbf{R}_{+}}$-adapted Lévy process on $\left(\Omega, \mathscr{F},\left(\widehat{\mathscr{F}}_{\theta}\right)_{\theta \in \mathbf{R}_{+}}, \mathbf{P}\right)$ with Law $(\widehat{L})=$ Law $(X)$ and

2) $M=\left(\widehat{L}_{\widehat{T}_{t}}\right)_{t \in \mathbf{R}_{+}}$.

If we define the $\left(\Omega, \mathscr{F},\left(\mathscr{F}_{t}\right)_{t \in \mathbf{R}_{+}}, \mathbf{P}\right)$-time change $\left(T_{\theta}\right)_{\theta \in \mathbf{R}_{+}}$by $T_{\theta}:=\inf \left\{t \in \mathbf{R}_{+}\right.$: $\left.\int_{0}^{t}\left|H_{s}\right|^{\alpha} d s>\theta\right\}$, then we may choose $\widehat{\mathscr{F}}_{\theta}=\mathscr{F}_{T_{\theta}}, \widehat{L}_{\theta}=M_{T_{\theta}}$ and $\widehat{T}$ as the inverse time change of $T$. In particular, $\widehat{T}_{t}=\int_{0}^{t}\left|H_{s}\right|^{\alpha} d s$ for $t \in \mathbf{R}_{+}$.

P r o of. First step. Obviously, $\left(T_{\theta}\right)_{\theta \in \mathbf{R}_{+}}$is a finite time change (cf. [4, I.1.28]). Choose $A_{t}=t$ for any $t \in \mathbf{R}_{+}$. Let $q \in \mathbf{Q}_{+}$and $\tau_{q}:=\inf \left\{t \geqslant q: \int_{q}^{t}\left|H_{s}\right|^{\alpha} d s>0\right\}$. Note that $H=0 A$-almost everywhere on $\left[q, \tau_{q}\right]$, which implies that $(\mathbf{P} \otimes A)\left(\left[q, \tau_{q}\right]\right)=0$. By Lemma $3,1_{\left[q, \tau_{q}\right]} \cdot M=\left(1_{\left[q, \tau_{q}\right]} H\right) \cdot X$ is a semimartingale with characteristics $(0,0,0)$ and hence it is 0 up to indistinguishability. Therefore, $M$ is constant on $\left[q, \tau_{q}\right]$ outside some P-null set. Since $\left(T_{\theta-}, T_{\theta}\right]=\cup\left\{\left[q, \tau_{q}\right]: q \in \mathbf{Q}_{+}\right.$with $\left.T_{\theta-}<q \leqslant T_{\theta}\right\}$, we have that $M$ is constant on any interval $\left(T_{\theta-}, T_{\theta}\right], \theta \in \mathbf{R}_{+}$. By right-continuity, it follows

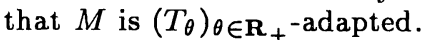

Second step. Let $U \in L(X)$. The cumulant process of $X$ is of the form $\mathscr{K}^{X}(U)=$ $\kappa(U) \cdot A$, where $\kappa(\cdot)$ is given by $\kappa(u)=\gamma \int\left(e^{i u x}-1-i u x 1_{[-1,1]}(x)\right)|x|^{-(1+\alpha)} d x$ for $\alpha \neq 2$ and $\kappa(u)=-\gamma u^{2}$ for $\alpha=2$ (with some constant $\gamma \in \mathbf{R}_{+}$, cf., e.g., [8, p. 6] and Lemma 4). Straightforward calculations show that $\kappa(u v)=|v|^{\alpha} \kappa(u)$ for any $u, v \in \mathbf{R}$.

Third step. Let $u \in \mathbf{R}$. If $\mathscr{K}^{M}, \mathscr{K}^{\widehat{L}}$ denote the cumulant processes of $M$ and $\widehat{L}$, respectively, Lemmas 2 and 5 yield that $\mathscr{K}^{M}(u)=\mathscr{K}^{X}(u H)$ and $\mathscr{K}^{\widehat{L}}(u)_{\theta}=\mathscr{K}^{M}(u)_{T_{\theta}}$. Together, it follows that

$$
\begin{aligned}
\mathscr{K}^{\widehat{L}}(u)_{\theta} & =\mathscr{K}^{M}(u)_{T_{\theta}}=\mathscr{K}^{X}(u H)_{T_{\theta}}=\kappa(u H) \cdot A_{T_{\theta}}=\kappa(u)|H|^{\alpha} \cdot A_{T_{\theta}} \\
& =\kappa(u) \int_{0}^{T_{\theta}}\left|H_{s}\right|^{\alpha} d s=\kappa(u) \theta=\mathscr{K}^{X}(u)_{\theta}
\end{aligned}
$$

for any $\theta \in \mathbf{R}_{+}$. From Lemma 4 and [4, II.2.42, II.4.19], it follows that $\widehat{L}$ is a Lévy process with the same distribution as $X$.

Fourth step. By $[2,(10.2)]$ and Lemma $6, \widehat{T}$ is a finite time change and $\widehat{T}_{t}=$

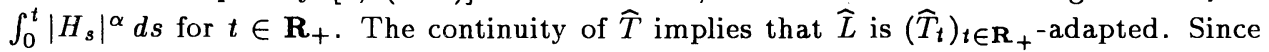
$t \in\left[T_{\widehat{T}_{t-}}, T_{\widehat{T}_{t}}\right]$ and $M$ is $\left(T_{\theta}\right)_{\theta \in \mathbf{R}_{+}}$-adapted, it follows that $\widehat{L}_{\widehat{T}_{t}}=M_{(T \circ \widehat{T})_{t}}=M_{t}$ for $t \in \mathbf{R}_{+}$. 
If we consider only nonnegative integrands $H$, we can extend the statement to asymmetric $\alpha$-stable Lévy motions (including deterministic linear functions).

Theorem 3. Let $X$ be an $\alpha$-stable Lévy motion. Moreover, let $M:=H \cdot X$ for some nonnegative $H \in L(X)$ such that $\int_{0}^{t} H_{s}^{\alpha} d s \rightarrow \infty$ for $t \rightarrow \infty$. Then there exist a filtration $\left(\widehat{\mathscr{F}}_{\theta}\right)_{\theta \in \mathbf{R}_{+}}$on $(\Omega, \mathscr{F})$, a process $\left(\widehat{L}_{\theta}\right)_{\theta \in \mathbf{R}_{+}}$, and a finite time change $\left(\widehat{T}_{t}\right)_{t \in \mathbf{R}_{+}}$such that

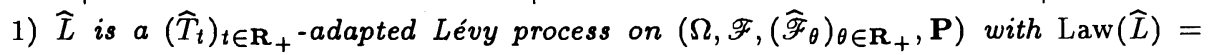
$\operatorname{Law}(X)$ and

2) $M=\left(\widehat{L}_{\widehat{T}_{t}}\right)_{t \in \mathbf{R}_{+}}$.

If we define the $\left(\Omega, \mathscr{F},\left(\mathscr{F}_{t}\right)_{t \in \mathbf{R}_{+}}, \mathbf{P}\right)$-time change $\left(T_{\theta}\right)_{\theta \in \mathbf{R}_{+}}$by $T_{\theta}:=\inf \left\{t \in \mathbf{R}_{+}\right.$: $\left.\int_{0}^{t} H_{s}^{\alpha} d s>\theta\right\}$, then we may choose $\widehat{\mathscr{F}}_{\theta}=\mathscr{F}_{T_{\theta}}, \widehat{L}_{\theta}=M_{T_{\theta}}$ and $\widehat{T}$ as the inverse time change of $T$. In particular, $\widehat{T}_{t}=\int_{0}^{t} H_{s}^{\alpha} d s$ for $t \in \mathbf{R}_{+}$.

$\mathrm{Pr}$ o of. This is shown along the same lines as the previous theorem. Only the second step has to be modified slightly. Here,

$$
\begin{aligned}
\kappa(u)= & \gamma_{1} \int 1_{(0, \infty)}(x)\left(e^{i u x}-1\right) x^{-(1+\alpha)} d x \\
& +\gamma_{2} \int 1_{(-\infty, 0)}(x)\left(e^{i u x}-1\right)|x|^{-(1+\alpha)} d x, \quad \alpha<1, \\
\kappa(u)= & i u \mu+\gamma_{1} \int\left(e^{i u x}-1-i u x 1_{[-1,1]}(x)\right)|x|^{-(1+\alpha)} d x, \quad \alpha=1, \\
\kappa(u)= & \gamma_{1} \int 1_{(0, \infty)}(x)\left(e^{i u x}-1-i u x\right) x^{-(1+\alpha)} d x \\
& +\gamma_{2} \int 1_{(-\infty, 0)}(x)\left(e^{i u x}-1-i u x\right)|x|^{-(1+\alpha)} d x, \quad 1<\alpha<2, \\
\kappa(u)= & -\gamma_{1} u^{2}, \quad \alpha=2
\end{aligned}
$$

(with some constants $\gamma_{1}, \gamma_{2} \in \mathbf{R}_{+}, \mu \in \mathbf{R}$, cf. [9, Theorem 14.7] or [10, III.1c, Theorem 3]). Again, straightforward calculations show that $\kappa(u v)=v^{\alpha} \kappa(u)$ for any $u, v \in \mathbf{R}_{+}$.

The following result shows that there are no other Lévy processes such that an analogous statement holds.

Theorem 4. Suppose that $X$ is a real-valued Lévy process with the following property: For any $H \in \mathbf{R} \backslash\{0\}$ there exist a filtration $\left(\widehat{\mathscr{F}}_{\theta}\right)_{\theta \in \mathbf{R}_{+}}$on $(\Omega, \mathscr{F})$, a process $\left(\widehat{L}_{\theta}\right)_{\theta \in \mathbf{R}_{+}}$, and a finite time change $\left(\widehat{T}_{t}\right)_{t \in \mathbf{R}_{+}}$such that

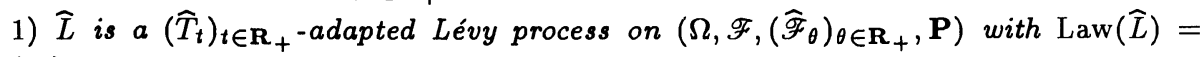
Law $(X)$ and

2) $H X=\left(\widehat{L}_{\widehat{T}_{t}}\right)_{t \in \mathbf{R}_{+}}$.

Then $X$ is a symmetric $\alpha$-stable Lévy motion.

If we claim the above property only for any $H \in(0, \infty)$, it follows that $X$ is an $\alpha$-stable Lévy motion.

P r o o f. First step. Let $H \in(0, \infty)$ and $u \in \mathbf{R}$. Note that the cumulant processes of Levy processes are deterministic, linear in time, and characterized by the Lévy-Khinchine triplet. By Lemmas 2 and 5, the cumulant process of $H X$ is of the form

$$
\kappa(u H) A_{t}=\mathscr{K}^{X}(u H)_{t}=\mathscr{K}^{H X}(u)_{t}=\mathscr{K}^{\widehat{L}}(u)_{\widehat{T}_{t}}=\kappa(u) A_{\widehat{T}_{t}}
$$

if we set $A_{t}=t$ and $\mathscr{K}^{X}(u)=\kappa(u) \cdot A$ denotes the cumulant process of $X$ and $\widehat{L}$. The process $\left(A_{\widehat{T}_{t}}\right)_{t \in \mathbf{R}_{+}}$can be written as $A_{\widehat{T}_{t}}=a \cdot A_{t}+N_{t}$ for some $\left(\mathscr{F}_{t}\right)_{t \in \mathbf{R}_{+}}$-predictable, nonnegative process $a$ and some increasing process $N$ which is orthogonal to Lebesgue measure (cf. [4, I.3.13]). Since the left-hand side of equation (1) is absolutely continuous with respect to Lebesgue measure, it follows that $\kappa(u H) \cdot A=(\kappa(u) a) \cdot A$, which implies that $\kappa(u H)$ is a multiple of $\kappa(u)$, say $\kappa(u H)=f(H) \kappa(u)$ with some function $f:(0, \infty) \rightarrow$ $(0, \infty)$. Note that $f\left(H H^{\prime}\right)=f(H) f\left(H^{\prime}\right)$ and $f$ is continuous because of the continuity 
of $\kappa$. Therefore, $\ln (f(\exp (\cdot)))$ is a linear function, hence $f(H)=H^{\alpha}$ for some $\alpha \in \mathbf{R}$. Note that $\alpha=0$ is only possible for $\kappa=0$, in which case $X=0$. Suppose that $\alpha \neq 0$.

Second step. Let $k_{1}, k_{2} \in(0, \infty)$. Since $u \mapsto \exp (\kappa(u))$ is the characteristic function of $X_{1}$, we have that

$$
\begin{aligned}
\left(P^{k_{1} X_{1}} * P^{k_{2} X_{1}}\right)^{\wedge}(u) & =\exp \left(\kappa\left(u k_{1}\right)+\kappa\left(u k_{2}\right)\right)=\exp \left(\left(k_{1}^{\alpha}+k_{2}^{\alpha}\right) \kappa(u)\right) \\
& =\exp \left(\kappa\left(u\left(k_{1}^{\alpha}+k_{2}^{\alpha}\right)^{-\alpha}\right)\right)=\left(P^{\left(k_{1}^{\alpha}+k_{2}^{\alpha}\right)^{-\alpha} X_{1}}\right)^{\wedge}(u),
\end{aligned}
$$

where $\left(P^{Y}\right)^{\wedge}$ denotes the characteristic function of $Y$. It follows that $X_{1}$ is strictly stable (cf. [8, Definition 1.1.1]).

Third step. If the property in the theorem holds for negative $H$, the same reasoning as above yields that $\kappa(u)=f(-1) \kappa(-u)$ for some $f(-1) \in \mathbf{R}$ that does not depend on $u$. From $\kappa(u)=f(-1) \kappa(-u)=(f(-1))^{2} \kappa(u)$ it follows that $f(-1)=1$. This implies that $\left(P^{X_{1}}\right)^{\wedge}(u)=\left(P^{X_{1}}\right)^{\wedge}(-u)$, which in turn yields that $\operatorname{Law}\left(X_{1}\right)=\operatorname{Law}\left(-X_{1}\right)$. Hence, $X_{1}$ is symmetric stable.

It is an open question whether the previous theorem still holds if we remove the constraint that $\widehat{L}$ is $\left(\widehat{T}_{t}\right)_{t \in \mathbf{R}_{+}}$-adapted.

\section{Tools from stochastic calculus.}

Lemma 1. Let $Z$ be a semimartingale such that $Z, Z_{-}$are $(\mathbf{C} \backslash\{0\})$-valued. Then there exists an up to indistinguishability unique $\mathbf{C}$-valued semimartingale $X$ such that $X_{0}=0$ and $Z=Z_{0} \mathscr{E}(X)$. It is given by $X=\left(1 / Z_{-}\right) \cdot Z$.

$\mathrm{P} \mathrm{r}$ o o $\mathrm{f}$. The existence and explicit form of $X$ follows from $1+\left(Z_{-} / Z_{0}\right) \cdot\left(\left(1 / Z_{-}\right) \cdot\right.$ $Z)=1+\left(Z_{-}\left(1 / Z_{-}\right)\right) \cdot Z / Z_{0}=Z / Z_{0}$. Now let $X$ be any semimartingale with $X_{0}=0$ and $Z=Z_{0} \mathscr{E}(X)$. Then $X=\left(\left(1 / Z_{-}\right) Z_{-}\right) \cdot X=\left(1 / Z_{-}\right) \cdot\left(Z_{-} \cdot X\right)=\left(1 / Z_{-}\right) \cdot Z$, which yields the uniqueness.

$\mathrm{D}$ e $\mathrm{f}$ i $\mathrm{n}$ i t i o $\mathrm{n} 3$. We call the process $X$ in the previous lemma stochastic logarithm of $Z$ and write $\mathscr{L}(Z):=X$.

Let $X$ be a $\mathbf{R}^{d}$-valued semimartingale whose characteristics $(B, C, \nu)$ relative to some truncation function $h: \mathbf{R}^{d} \rightarrow \mathbf{R}^{d}$ are given in the form $B=b \cdot A, C=c \cdot A, \nu=$ $A \otimes F$, where $A \in \mathscr{A}_{1 \text { oc }}^{+}$is a predictable process, $b$ is a predictable $\mathbf{R}^{d}$-valued process, $c$ is a predictable $\mathbf{R}^{d \times d}$-valued process whose values are nonnegative, symmetric matrices, and $F$ is a transition kernel from $\left(\Omega \times \mathbf{R}_{+}, \mathscr{P}\right)$ into $\left(\mathbf{R}^{d}, \mathscr{B}^{d}\right)$. By [4, Proposition II.2.9] such a representation always exists.

$\mathrm{D}$ e fin it i o $\mathrm{n}$ 4. Suppose $\mathbf{R}^{d}$-valued process $U \in L(X)$. The cumulant process $\mathscr{K}^{X}(U)$ of $X$ in $U$ is defined as the predictable part of finite variation in the canonical decomposition of the special semimartingale $\mathscr{L}\left(\exp \left(i U^{\top} \cdot X\right)\right.$ ) (in the sense of $[4$, I.4.22]).

$\mathrm{R}$ e $\mathrm{m}$ a $\mathrm{r}$ k. Note that $\exp \left(i U^{\top} \cdot X\right)$ is bounded and hence is a complex special semimartingale. Therefore, $\mathscr{L}\left(\exp \left(i U^{\top} \cdot X\right)\right)=\exp \left(-i U^{\top} \cdot X_{-}\right) \cdot \exp \left(i U^{\top} \cdot X\right)$ is a special semimartingale as well (cf. [2,(2.51)]).

Lemma 2. Let $H \in L(X)$ and $U \in L\left(H^{\top} \cdot X\right)$. Then $\mathscr{K}^{H^{\top} \cdot X}(U)=\mathscr{K}^{X}(U H)$.

P r o o f. From $H \in L(X), U \in L\left(H^{\top} \cdot X\right)$ ), it follows that $U H \in L(X)$ (cf. [1, Proposition 5.1]). The assertion is now readily obtained from $\exp \left(i U \cdot\left(H^{\top} \cdot X\right)\right)=$ $\exp \left(i(U H)^{\top} \cdot X\right)$.

Lemma 3. Let $H \in L(X)$. Then the characteristics $(\widetilde{B}, \tilde{C}, \widetilde{\nu})$ of $H^{\top} \cdot X$ relative to some truncation function $h_{1}: \mathbf{R} \rightarrow \mathbf{R}$ are of the form $\widetilde{B}=\tilde{b} \cdot A, \widetilde{C}=\tilde{c} \cdot A, \widetilde{\nu}=A \otimes \widetilde{F}$, where

$$
\begin{aligned}
\tilde{b}_{t} & =H_{t}^{\top} b_{t}+\int\left(h_{1}\left(H_{t}^{\top} x\right)-H_{t}^{\top} h(x)\right) F_{t}(d x), \\
\tilde{c}_{t} & =H_{t}^{\top} c_{t} H_{t}, \\
\tilde{F}_{t}(G) & =\int 1_{G}\left(H_{t}^{\top} x\right) F_{t}(d x) \quad \text { for any } G \in \mathscr{B} \text { with } 0 \notin G .
\end{aligned}
$$


$\mathrm{Pr}$ o o f. For locally bounded integrands the lemma is proved in [4, IX.5.3]. Since $H \in L_{\text {loc }}^{1}\left(X^{c}\right)$, it follows that $\widetilde{C}=\left\langle H^{\top} \cdot X^{c}, H^{\top} \cdot X^{c}\right\rangle=\left(H^{\top} c H\right) \cdot A$ (cf. [1, Proposition 5.2] and $[3,1 \mathrm{a}])$. Moreover, $\Delta\left(H^{\top} \cdot X\right)=H^{\top} \Delta X$ implies that $1_{G}(x) * \mu^{H^{\top} \cdot X}=1_{G}\left(H^{\top} x\right) * \mu^{X}$ and hence $1_{G}(x) * \widetilde{\nu}=1_{G}\left(H^{\top} x\right) * \nu$ for any $G \in \mathscr{B}$ with $0 \notin G$. By [1, Proposition 5.2], there exist a set $\Delta \in \mathscr{P} \otimes \mathscr{B}^{d}$ and a predictable process $\bar{B} \in \mathscr{V}^{d}$ such that

$$
\begin{aligned}
X & =X_{0}+X^{c}+x 1_{\Delta C}(x) *\left(\mu^{X}-\nu\right)+x 1_{\Delta}(x) * \mu^{X}+\bar{B} \\
H^{\top} \cdot X & =H^{\top} \cdot X^{c}+H^{\top} x 1_{\Delta} C(x) *\left(\mu^{X}-\nu\right)+H^{\top} x 1_{\Delta}(x) * \mu^{X}+H^{\top} \cdot \bar{B} .
\end{aligned}
$$

Since $X$ can also be written in its canonical semimartingale representation $X=X_{0}+$ $X^{c}+h(x) *\left(\mu^{X}-\nu\right)+(x-h(x)) * \mu^{X}+B$, it follows from [1, Proposition 5.3] that $\bar{B}=B+\left(x 1_{\Delta C}(x)-h(x)\right) * \nu=\left(b+\int\left(x 1_{\Delta C}(x)-h(x)\right) F(d x)\right) \cdot A$. Similarly, the canonical semimartingale representation of $H^{\top} \cdot X$ equals $H^{\top} \cdot X=H^{\top} \cdot X^{c}+h_{1}\left(H^{\top} x\right) *$ $\left(\mu^{X}-\nu\right)+\left(H^{\top} x-h_{1}\left(H^{\top} x\right)\right) * \mu^{X}+\widetilde{B}$, which yields, again using [1, Proposition 5.3], that

$$
\begin{aligned}
\widetilde{B}= & H^{\top} \cdot \bar{B}+\left(h_{1}\left(H^{\top} x\right)-H^{\top} x 1_{\Delta^{C}}(x)\right) * \nu \\
= & \left(H^{\top}\left(b+\int\left(x 1_{\Delta^{C}}(x)-h(x)\right) F(d x)\right)\right) \cdot A \\
& +\left(\int\left(h_{1}\left(H^{\top} x\right)-H^{\top} x 1_{\Delta^{C}}(x)\right) F(d x)\right) \cdot A \\
= & \left(H^{\top} b+\int\left(h_{1}\left(H^{\top} x\right)-H^{\top} h(x)\right) F(d x)\right) \cdot A .
\end{aligned}
$$

This proves the assertion.

Lemma 4. Let $U \in L(X)$. Then $\mathscr{K}^{X}(U)=\kappa(U) \cdot A$, where

$$
\kappa(U)_{t}=i U_{t}^{\top} b_{t}-\frac{1}{2} U_{t}^{\top} c_{t} U_{t}+\int\left(e^{i U_{t}^{\top} x}-1-i U_{t}^{\top} h(x)\right) F_{t}(d x) .
$$

P r o o f. First step. From Lemmas 2 and 3 it follows that $\mathscr{K}^{X}(U)=\mathscr{K}^{U^{\top} \cdot X}(1)$ and $\kappa(U)=\kappa^{U^{\top} \cdot X}(1)$, where $\kappa^{U^{\top} \cdot X}$ is defined as $\kappa$ but for the process $U^{\top} \cdot X$ instead of $X$. Therefore, it suffices to prove the statement for $\mathbb{R}^{1}$-valued $X$ with $X_{0}=0$ and $U=1$.

Second step. Let $Y:=i X$. Applying Itô's formula (e.g. as in [1, Lemma 5.5]) yields that $\exp (i X)=\exp (Y)=\mathscr{E}\left(Y+\frac{1}{2}\left\langle Y^{c}, Y^{c}\right\rangle+\left(e^{x}-1-x\right) * \mu^{Y}\right)$ and hence $L:=$ $\mathscr{L}(\exp (i X))=Y+\frac{1}{2}\left\langle Y^{c}, Y^{c}\right\rangle+\left(e^{x}-1-x\right) * \mu^{Y}$. Note that $Y=i\left(B+X^{c}+h(x) *\right.$ $\left.\left(\mu^{X}-\nu\right)+(x-h(x)) * \mu^{X}\right)$. Together, we obtain that

$$
L=i B+i X^{c}+i h(x) *\left(\mu^{X}-\nu\right)-\frac{1}{2}\left\langle X^{c}, X^{c}\right\rangle+\left(e^{i x}-1-i h(x)\right) * \mu^{X} .
$$

On the other hand, the canonical decomposition of $L$ is $L=V+L^{c}+x *\left(\mu^{L}-\nu^{L}\right)=$ $V+L^{c}+\left(e^{i x}-1\right) *\left(\mu^{X}-\nu\right)$, where $V \in \mathscr{V}$ is predictable. From [1, Proposition 5.3] it follows that $V=i B-\frac{1}{2} C+\left(e^{i x}-1-i h(x)\right) * \nu=\kappa(1) \cdot A$. Therefore $\mathscr{X}^{\bar{X}}(1)=\kappa(1) \cdot A$ as claimed.

$\mathrm{R}$ e m a r k. In particular, the cumulant process $\mathscr{K}^{X}(U)$ for $U \in L(X)$ can be obtained from the cumulant processes $\mathscr{K}^{X}(u), u \in \mathbb{R}^{d}$. Also note that by $[4$, II.2.42] the cumulant processes $\mathscr{K}^{X}(u), u \in \mathbb{R}^{d}$, uniquely determine the characteristics $(B, C, \nu)$ of $X$.

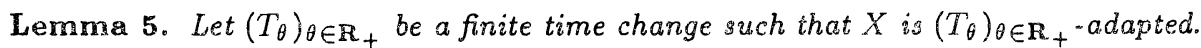
Then the process $\left(\widehat{X}_{\theta}\right)_{\theta \in \mathbb{R}_{+}}$defined by $\widehat{X}_{\theta}:=X_{T_{\theta}}$ is a semimartingale on $\left(\Omega, \mathscr{F},\left(\widehat{\mathscr{F}}_{\theta}\right)_{\theta \in \mathbf{R}_{+}}, \mathbf{P}\right)$ whose characteristics $(\widehat{B}, \widehat{C}, \widehat{\nu})$ are of the form

$$
\widehat{B}_{\theta}=B_{T_{\theta}}, \quad \widehat{C}_{\theta}=C_{T_{\theta}}, \quad I_{G} * \widehat{\nu}_{\theta}=I_{G} * \nu_{T_{\theta}}
$$


for $\theta \in \mathbf{R}_{+}, G \in \mathscr{B}^{d}$ with $0 \notin G$. Moreover, its cumulant process satisfies

$$
\mathscr{K}^{\widehat{X}}(u)_{\theta}=\mathscr{K}^{X}(u)_{T_{\theta}} \quad \text { for } \theta \in \mathbf{R}_{+}, u \in \mathbf{R}^{d} .
$$

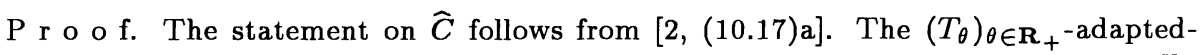
ness of $X$ implies that $1_{G} * \mu_{T_{\theta}-}^{X}=\sum_{t<T_{\theta}} 1_{G}\left(\Delta X_{t}\right)=\sum_{t \leqslant T_{\theta}} 1_{G}\left(\Delta X_{t}\right)=1_{G} * \mu_{T_{\theta}}^{X}$, hence $\mu^{X}$ is $\left(T_{\theta}\right)_{\theta \in \mathbf{R}_{+}}$-adapted in the sense of $[2,(10.25)]$. Since $\Delta \widehat{X}_{\theta} 1_{\left\{\Delta \widehat{X}_{\theta} \neq 0\right\}}=$ $\Delta X_{T_{\theta}-1}\left\{\Delta X_{\left.T_{\theta}-\neq 0\right\}}\right.$, we have that $1_{G} * \mu_{\theta}^{\widehat{X}}=\sum_{\tilde{\theta} \leqslant \theta} 1_{G}\left(\Delta \widehat{X}_{\tilde{\theta}}\right)=\sum_{t<T_{\theta}} 1_{G}\left(\Delta X_{t}\right)=$ $1_{G} * \mu_{T_{\theta}}^{X}$ for $\theta \in \mathbf{R}_{+}, G \in \mathscr{B}^{d}$ with $0 \notin G$. It follows from [2, (10.27)] that $1_{G} * \widehat{\nu}_{\theta}=1_{G} * \nu_{T_{\theta}}$. Moreover, we have $\widehat{X}_{\theta}-(x-h(x)) * \mu_{\theta}^{\widehat{X}}=X_{T_{\theta}}-(x-h(x)) * \mu_{T_{\theta}}^{X}$. Therefore, the statement on $\widehat{B}$ follows from $[2,(10.17) b]$. Finally, observe that by Lemma 4 ,

$$
\begin{aligned}
\mathscr{K}^{\widehat{X}}(u)_{\theta} & =i u^{\top} \widehat{B}_{\theta}-\frac{1}{2} u^{\top} \widehat{C}_{\theta} u+\left(e^{i u^{\top} x}-1-i u^{\top} h(x)\right) * \widehat{\nu}_{\theta} \\
& =i u^{\top} B_{T_{\theta}}-\frac{1}{2} u^{\top} C_{T_{\theta}} u+\left(e^{i u^{\top} x}-1-i u^{\top} h(x)\right) * \nu_{T_{\theta}}=\mathscr{K}^{X}(u)_{T_{\theta}} .
\end{aligned}
$$

$\mathrm{R} \mathrm{e} \mathrm{m} \mathrm{a} \mathrm{r} \mathrm{k.} \mathrm{Even} \mathrm{though} \mathrm{the} \mathrm{semimartingale} \mathrm{property} \mathrm{is} \mathrm{preserved} \mathrm{under} \mathrm{arbitrary}$ finite time changes, Lemma 5 generally ceases to hold. For example, even a continuous pro-

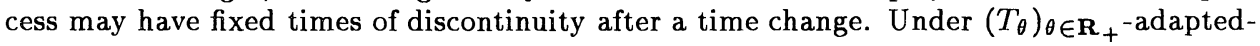
ness, however, many properties are preserved (cf. [2, Chapter X]).

We end with a characterisation of $L(X)$ for $\alpha$-stable Lévy motions $X$ which can be found in [5] (cf. also [7]). We give a more direct proof here.

Lemma 6. Let $X$ be a nonconstant $\alpha$-stable Lévy motion and $H$ a predictable realvalued process. Then $H \in L(X)$ if and only if $\int_{0}^{t}\left|H_{s}\right|^{\alpha} d s<\infty \mathbf{P}$-almost surely for any $t \in \mathbf{R}_{+}$.

P r o of. Since $H=H^{\prime}+H^{\prime \prime}$ with $H_{t}^{\prime}:=H_{t} 1_{\left\{\left|H_{t}\right|>1\right\}}+1_{\left\{\left|H_{t}\right| \leqslant 1\right\}}$ and $H_{t}^{\prime \prime}:=$ $\left(H_{t}-1\right) 1_{\left\{\left|H_{t}\right| \leqslant 1\right\}}$, it suffices to consider the case $|H| \geqslant 1$. By $(B, C, \nu)$ we denote the characteristics of $X$ relative to $h: \mathbf{R} \rightarrow \mathbf{R}$, i.e., $B_{t}=b t, C_{t}=c t, \nu=\lambda \otimes F$, where $(b, c, F)$ is the Lévy-Khinchine triplet of Law $\left(X_{1}\right)$. By [1, Proposition 5.2], $H \in L(X)$ holds if and only if $H \in L_{10 c}^{1}\left(X^{c}\right), H x 1_{\{|H x| \leqslant 1\}} \in G_{10 c}\left(\mu^{X}\right),\left|H x 1_{\{|H x|>1\}}\right| * \mu^{X} \in \mathscr{V}$, and $H \in L_{s}(\widetilde{B})$, where $\widetilde{B}=B+\left(x 1_{\{|H x| \leqslant 1\}}-h(x)\right) * \nu$. By $\alpha \in(0,2]$ we denote the index of stability of $X$.

Case 1. $\alpha=2$. In this case $\mu^{X}=0, B=0$, and $X$ is a continuous local martingale. Therefore, $H \in L(X)$ if and only if $H \in L_{\text {loc }}^{1}(X)$, i.e., if and only if $H^{2} \cdot\langle X, X\rangle \in \mathscr{V}$. Since $\langle X, X\rangle_{t}=c t$, the claim follows.

Case 2. $\alpha \neq 2$. Note that $X^{c}=0$. Moreover, $\left|H x 1_{\{|H x|>1\}}\right| * \mu^{X} \leqslant\left|H x 1_{\{|x|>1\}}\right| *$ $\mu^{X} \in \mathscr{V}$ holds automatically because $\left|H x 1_{\{|x|>1\}}\right| * \mu_{t}^{X}$ is a finite sum for fixed $t$. By [4, II.1.33c], $H x 1_{\{|H x| \leqslant 1\}} \in G_{\text {loc }}\left(\mu^{X}\right)$ if and only if $H^{2} x^{2} 1_{\{|H x| \leqslant 1\}} * \nu \in \mathscr{V}$. But note that $\nu=\lambda \otimes F$ and $\int H_{t}^{2} x^{2} 1_{\left\{\left|H_{t} x\right| \leqslant 1\right\}} F(d x)=\left|H_{t}\right|^{\alpha} \int y^{2} 1_{\{|y| \leqslant 1\}} F(d y)$ because the density of $F$ is proportionate to $|x|^{-(1+\alpha)}$ on $(0, \infty)$ (respectively $(-\infty, 0)$ ). Since $\int y^{2} 1_{\{|y| \leqslant 1\}} F(d y)<\infty$, it follows that $H^{2} x^{2} 1_{\{|H x| \leqslant 1\}} * \nu \in \mathscr{V}$ if and only if $F=0$ or $\int_{0}^{\cdot}\left|H_{t}\right|^{\alpha} d t \in \mathscr{V}$. For the drift part, we have to distinguish several cases.

Firstly, let $\alpha=1$. If we choose a symmetric $h$, then $\left(x 1_{\{|H| x \mid \leqslant 1\}}-h(x)\right) * \nu=0$ because $F$ is symmetric. Therefore, $\widetilde{B}=B$ is a deterministic, linear function. This implies that $H \in L_{s}(\widetilde{B})$ if and only if $\widetilde{B}=0$ or $\int_{0}\left|H_{t}\right| d t \in \mathscr{V}$.

Secondly, let $\alpha \in(1,2)$. By [9, Theorem 14.7], we can use $h(x)=x$ and have $B=0$ for this choice of $h$. Consequently, $\widetilde{B}=-x 1_{\{|H|>\mid>1\}} * \nu$. Therefore, $H \in L_{s}(\widetilde{B})$ holds if $|H x| 1_{\{|H x|>1\}} * \nu \in \mathscr{V}$. Since $\int|y| 1_{\{|y|>1\}} F(d y)<\infty$, a similar reasoning as above yields that $|H x| 1_{\{|H x|>1\}} * \nu \in \mathscr{V}$ if and only if $F=0$ or $\int_{0}^{\cdot}\left|H_{t}\right|^{\alpha} d t \in \mathscr{V}$.

Finally, let $\alpha \in(0,1)$. By [9, Theorem 14.7], we can use $h(x)=0$ and have $B=0$ for this choice of $h$. Therefore, $\widetilde{B}=x 1_{\{|H| \leqslant \mid \leqslant 1\}} * \nu$ in this case. Note that $\int|y| 1_{\{|y| \leqslant 1\}} F(d y)<\infty$. As for $\alpha>1$, it follows that $H \in L_{s}(\widetilde{B})$ holds if $\int_{0}^{\cdot}\left|H_{t}\right|^{\alpha} d t \in \mathscr{V}$. 


\title{
REFERENCES
}

1. Goll T., Kallsen J. Optimal portfolios for logarithmic utility. - Stochastic Process. Appl., 2000, v. 89, p. 31-48.

2. Jacod J. Calcul stochastique et problèmes de martingales. Berlin-Heidelberg: Springer-Verlag, 1979, 539 p.

3. Jacod $J$. Intégrales stochastiques par rapport à une semi-martingale vectorielle et changements de filtration. - Lecture Notes in Math., 1980, v. 784, p. 161-172.

4. Jacod J., Shiryaev A. Limit Theorems for Stochastic Processes. Berlin-Heidelberg: Springer-Verlag, 1987. Рус. перев.: Жаход Ж., Ширяев A. Н. Предельные теоремы для случайных процессов. Т. 1, 2. М.: Физматлит, 1994, 544 с; 368 с.

5. Kallenberg $O$. Some time change representations of stable integrals, via predictable transformations of local martingales. - Stochastic Process. Appl., 1992, v. 40, № 2, p. 199-223.

6. Revuz D., Yor M. Continuous Martingales and Brownian Motion. Berlin: SpringerVerlag, 1999, $602 \mathrm{p}$.

7. Rosiński J., Woyczyński $W$. On Itô stochastic integration with respect to $p$-stable motion: Inner clock, integrability of sample paths, double and multiple integrals. Ann. Probab., 1986, v. 14, p. 271-286.

8. Samorodnitsky G., Taqqu M. Stable Non-Gaussian Random Processes: Stochastic Models with Infinite Variance. New York: Chapman\&Hall, 1994, 632 p.

9. Sato K. Lévy Processes and Infinitely Divisible Distributions. Cambridge: Cambridge Univ. Press, 1999, 486 p.

10. Ширяев $A$. Н. Основы стохастической финансовой математики. Т. 1, 2. М.: Фазис, 1998, 512 с.; 544 с. Англ. перев.: Shiryaev A. Essentials of Stochastic Finance. Singapore: World Sci. Publ., 1999.

Поступила в редакцию

4.V.2000

(C) $2001 \mathrm{r}$.

ХоХлов в. и.*

\section{МНОГОЧЛЕНЫ, ОРТОГОНАЛЬНЫЕ ОТНОСИТЕЛЬНО ПОЛИНОМИАЛЬНОГО РАСПРЕДЕЛЕНИЯ, И ФАКТОРИАЛЬНО-СТЕПЕННОЙ ФОРМАЛИЗМ}

\begin{abstract}
Ключевые слова и фразы: биномиальное распределение, пуассоновское распределение, полиномиальное распределение, многочлены Кравчука, многочлены Пуассона-Шарлье, факториально-степенные многочлены, ортогональные относительно полиномиального распределения многочлены, комбинаторные тождества, тождество Лерха.
\end{abstract}

Введение. Первоначальный вариант этого сообщения был направлен в редакцию журнала «Теория вероятностей и ее применения» еще в начале 1985 года. Однако в том варианте отсутствовали указания на иллюстрации таких применений полученных результатов и предложенной комбинаторной техники, которые могли бы представлять чисто вероятностный интерес. Поэтому автор решил в конечном счете ограничиться публикацией формулировок главных результатов в тезисах [6]. Впоследствии к ним были даны комментарии в докладе на IV Всемирном конгрессе Обшества им. Бернулли в Вене (август 1996 г.), а в докладе на Международном

* Математический институт им. В. А. Стеклова РАН, ул. Губкина, 8, 117966 Москва, ГСП-1, Россия; e-mail: tvp@tvp.ru 